\title{
MATHEMATICAL MODEL FOR ARTIFICIAL LEG USING ELECTRO-RHEOLOGICAL (ER) FLUID DAMPER
}

\section{El Arabi M. Mahmoud}

Lecturer, Department of Mechanical Engineering, Faculty of Engineering, Alexandria University, Egypt

(Received January 1, 2008 Accepted March 8, 2008)

\begin{abstract}
Walking is human's natural means to move from one place to another. Walking or gait cycle involves the motion of many of the body limbs and joints. Knee plays such an important role in the gait cycle. Motion of knee joint is not that simple kind of motion. It involves planer rolling and gliding motion as well as out of plane motion. Artificial limbs are used to enable amputees to function normally. Knee unit is a very critical component of the above knee prosthetic limb. .

In this work an artificial knee was designed consisting of a four bar linkage in which motion was controlled by a suitable Electro-rheological (ER) damper. A complete analysis of the kinematics of motion for the four bars and the ER damper was made. Stability moment required to control the artificial knee during walking was obtained, and comparison between the performance of artificial and normal knees was elaborated.
\end{abstract}

KEYWORDS: Human, Artificial, Electro- rheological, Mechanisms, Walking, Knees, Legs

\section{INTRODUCTION}

An artificial leg is required to function like a normal leg. It has to provide a means of support and ability of walking. The ideal artificial leg resembles a normal leg closely in how it allows the amputee to walk. The first artificial leg, the peg leg, caused a pole vaulting effect during walking. This was due to the user who has to raise the normal ankle to allow the peg leg to swing clear of the ground through the swing phase. Similarly the user tended to tilt the pelvis for the same reason. These actions caused bad walking styles, while consuming a lot of energy

Appoldt and Bennett [1] measured the loading on an above knee fiber-glass socket by building the socket with pressure transducers incorporated. Unfortunately their results are only accurate for the single socket used in the experiment. This is because all modern sockets have different geometries and external loadings due to differences in the amputees.

Bielefeldt and Schreck [2] found the difference in loading of four different sockets, during stance, for one patient. Their sockets were also built with transducers incorporated. From these results a general feel for the load paths can be gauged.

Dorious [3] investigated the dynamic response of the leg in torsion with application to snow ski injuries. A biomechanical model of the leg system was developed and injury mechanisms to the ankle, knee and tibia were presented, the 
objective was to determine how torsoional leg system dynamics influence injuries to the ankle, knee and tibia under impulse loading.

Rohrle and Sollbach [4] calculated the forces in the hip, knee and ankle joints during walking. The knowledge of the three dimensional movements of the human body and of the forces between foot and ground was a prerequisite.

Hunter [5] studied the behavior of the leg during oscillation by using a free oscillation method. The lower leg was represented by a simple mass, spring and dashpot model. Forty-five subjects underwent free vibration tests on their right legs. The force amplitude and the time period, for successive oscillations enabled calculation of the stiffness to mass ratio values .It was found that the time period for the second oscillation cycle was significantly lower than that of the first, implying a corresponding increase in stiffness.

Wen Liu and Nigg [6] reported that the simple spring damper mass models have been widely used to simulate human locomotion. However, most previous models have not accounted for the effect of non-rigid masses (wobbling masses) on impact forces. A simple mechanical model of the human body developed in this study considering the upper and lower bodies with each part represented by a rigid and wobbling mass. Spring damper units connected different masses to represent the rigid and wobbling masses.

Nisell [7] suggested that in turn the leg segmentation and the initial conditions may be a consequence of metabolic and bone stress constraints .In his study, the theoretical predictions were verified experimentally with respect to initial conditions and elastic joint characteristics in human running. Kinematic and kinetic variables were measured and the joint torques was estimated by inverse dynamics stiffness whereas elastic non-linearity describing the resulting joint characteristics were extracted from parameter fits. The results clearly supported the theoretical prediction and the knee joint was always stiffer and more extended than the ankle joint.

Various investigators (e.g. Hatze [8]; Kim and Pandy [9]) attempted to determine the passive elastic moments about the knee joint. Most of these studies differed in purpose and protocol and as a result much of the database remained incomplete and unclear.

Radcliffe [10] studied of the bio-mechanics of human walking, alignment principles, and methods of fitting for above-knee prostheses.

Notable examples were found in the work of Braune and Fischer [11] on human gait, and the publications of Schede [12] on scientific principles of fitting and alignment.

The objective of this study is to develop a theoretical leg model for use in an actively control algorithm using an Electro-rheological fluid damper. Before reaching this objective, force analysis has to be done, and dynamics in general of the artificial leg has also to be performed.

\section{DYNAMICS OF THE ARTIFICIAL LEG}

The suggested artificial leg Figure (1) consists of shank-foot and knee joint. The knee is represented by a four bar mechanism $\mathrm{ABCD}$, where its motion is controlled by Electro -rheological (ER) fluid damper. 


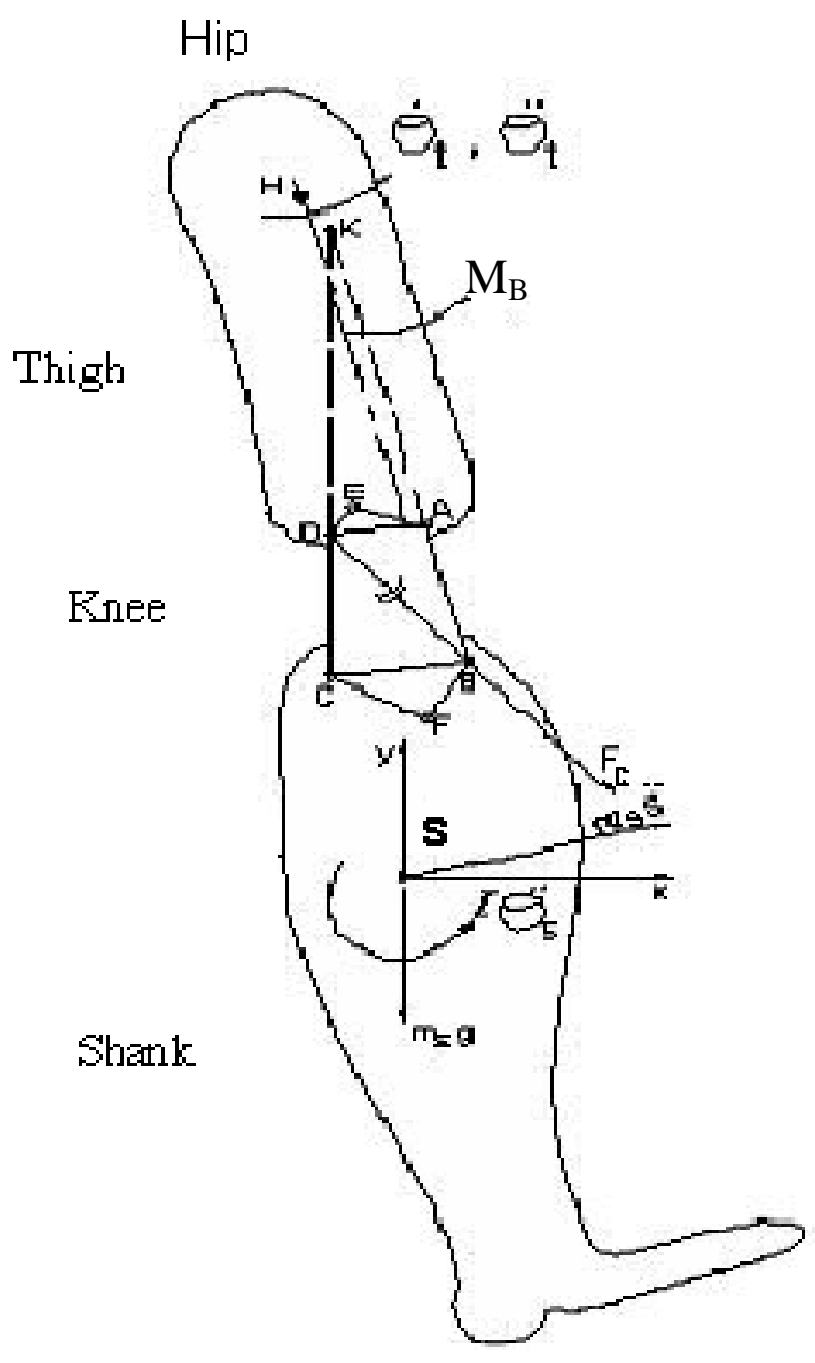

Fig.1 Schematic Representation of Artificial Leg

Figure (2) indicates the manner in which the rotation of knee center $(\mathrm{K})$, plotted when the moving socket changes its position during a full walking cycle. 


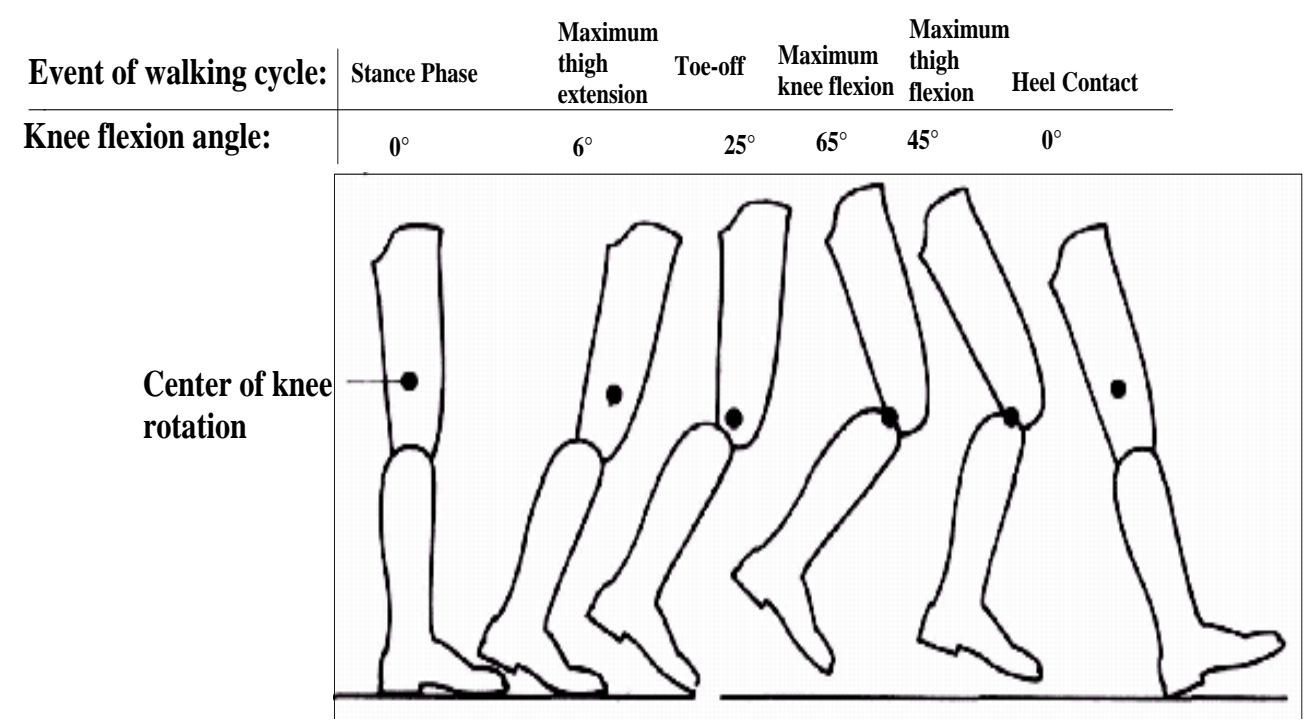

Fig.2 Change in Knee Center (K) During Walking [13]

In order to study the forces and moments acting on the artificial leg during walking Figure1, let us define the following parameters:

$\mathrm{F}_{\mathrm{c}}=$ Hydraulic controller force $(\mathrm{N})$

$\mathrm{e}=$ Unit vector in direction, (B-D)

$\mathrm{m}_{\mathrm{s}}=$ Mass of the shank $(\mathrm{kg})$

$\mathrm{g}=$ Gravitational acceleration $\left(\mathrm{m} / \mathrm{s}^{2}\right)$

$\mathrm{M}_{\mathrm{B}}=$ Stability moment $(\mathrm{N} \mathrm{m})$

$\mathrm{I}_{\mathrm{s}}=$ Mass moment of inertia of the shank, $\left(\mathrm{kg} \mathrm{m}^{2}\right)$

$\dot{\theta}_{\mathrm{s}}, \ddot{\theta}_{\mathrm{s}}=$ Angular velocity and angular acceleration of the shank, (rad $\left./ \mathrm{s}, \mathrm{rad} / \mathrm{s}^{2}\right)$

$\ddot{S}=$ Acceleration of the mass center of the shank $\left(\mathrm{m} / \mathrm{s}^{2}\right)$

Applying Newton second law and taking moments about the center of rotation $\mathrm{K}$, the following equations are obtained:

$$
\mathrm{M}_{\mathrm{B}}=\mathrm{I}_{\mathrm{s}} \ddot{\theta}_{\mathrm{s}}-(\overrightarrow{\mathrm{BK}}) \times \mathrm{F}_{\mathrm{C}}(\hat{\mathrm{e}})-(\overrightarrow{\mathrm{SK}}) \times \mathrm{m}_{\mathrm{s}}(\mathrm{g}-\ddot{\mathrm{S}})
$$

The acceleration of mass center of moving shank can be calculated in terms of acceleration of point $\mathbf{C}$, and kinematics variable $\dot{\theta}_{s}, \ddot{\theta}_{s}$ as:,

$$
\ddot{\mathrm{S}}=\ddot{\mathrm{C}}+\ddot{\theta}_{\mathrm{s}} \times(\overrightarrow{\mathrm{SC}})-\dot{\theta}_{s}^{2}(\overrightarrow{\mathrm{SC}})
$$


The acceleration of point $\mathrm{C}$ can be written as a function of link $\mathrm{CD}$ parameters and hip and thigh motions. This reads:

$$
\ddot{C}=\ddot{h}+\ddot{\theta}_{t} \times\left(\overrightarrow{D H)}+\dot{\theta}_{t}^{2} \overrightarrow{(D H}\right)+\ddot{\theta}_{c} \times(\overrightarrow{C D})-\dot{\theta}_{c}^{2}(\overrightarrow{C D})
$$

Where:

$\ddot{h}=$ Absolute acceleration of the hip joint, (point $\mathrm{H}$ ).

$\dot{\theta}_{t}, \ddot{\theta}_{t}=$ Absolute angular velocity and acceleration of the thigh.

$\dot{\theta}_{c}, \ddot{\theta}_{c}=$ Angular velocity and angular acceleration of the link CD,

$\dot{\theta}_{c}=\dot{\theta}_{t}+\omega_{3}$

$\ddot{\theta}_{c}=\ddot{\theta}_{t}+\alpha_{3}$

$\omega_{3}, \alpha_{3}=$ angular velocity and acceleration of link CD relative to AD.

The velocity of point $\mathrm{B}$ can be written as a function of link BA parameters and hip and thigh motion

$$
\begin{aligned}
& \dot{B}=\dot{A}+\dot{\theta}_{a} \times(\overrightarrow{A B}) \\
& \dot{A}=\dot{h}+\dot{\theta}_{t} \times(\overrightarrow{H A}) \\
& \dot{\mathrm{B}}, \dot{\mathrm{A}}=\text { velocity of points } \mathrm{B}, \mathrm{A} \\
& \dot{\theta}_{a}=\omega_{1}+\dot{\theta}_{t} \\
& \dot{\theta}_{a}=\text { angular velocity of link } \mathrm{AB} \\
& \omega_{1}=\text { angular velocity of link } \mathrm{AB} \text { relative to } \mathrm{AD} \\
& \dot{\mathrm{h}}=\text { absolute velocity of the hip joint (point } \mathrm{H} \text { ) }
\end{aligned}
$$

To find the stability moment $\left(\mathrm{M}_{\mathrm{B}}\right)$, analysis of four bar mechanism is required to obtain the velocities and accelerations during walking for artificial leg. Also a control force $F_{c}$ must be calculated according to a selected suitable model of (ER) damper.

\section{ANALYSIS OF FOUR BAR MECHANISM}

During swing phase the prosthesis swings in space about the knee center $\mathrm{K}$ as a compound pendulum. Consider a four bar mechanism $A B C D$, Figure (3) in which $A B$ $=\mathrm{a}, \mathrm{BC}=\mathrm{b}, \mathrm{CD}=\mathrm{c}$, and $\mathrm{DA}=\mathrm{d}$. The link $\mathrm{DA}$ has a fixed position. Let the links $\mathrm{AB}$, $\mathrm{BC}$, and $\mathrm{CD}$ make angles $\theta, \beta, \varphi$, along axis's of link DA.The link BD represents ER damper

The relation between the angles and link lengths may be developed by considering the links as vectors [14]. The expressions for displacement, velocity and acceleration analysis are derived below:

Let,

$\omega_{1}, \omega 2, \omega_{3}$ be the angular velocities of links $A B, C B, C D$ relative to $A D$

$\alpha_{1}, \alpha 2, \alpha_{3} \quad$ be the angular accelerations of links $\mathrm{AB}, \mathrm{CB}, \mathrm{CD}$ relative to $\mathrm{AD}$ 

$k_{3}=\frac{a^{2}-b^{2}+c^{2}+d^{2}}{2 a c}$
$k_{2}=\frac{d}{c}$
$k_{1}=\frac{d}{a}$

Center of knee rotation

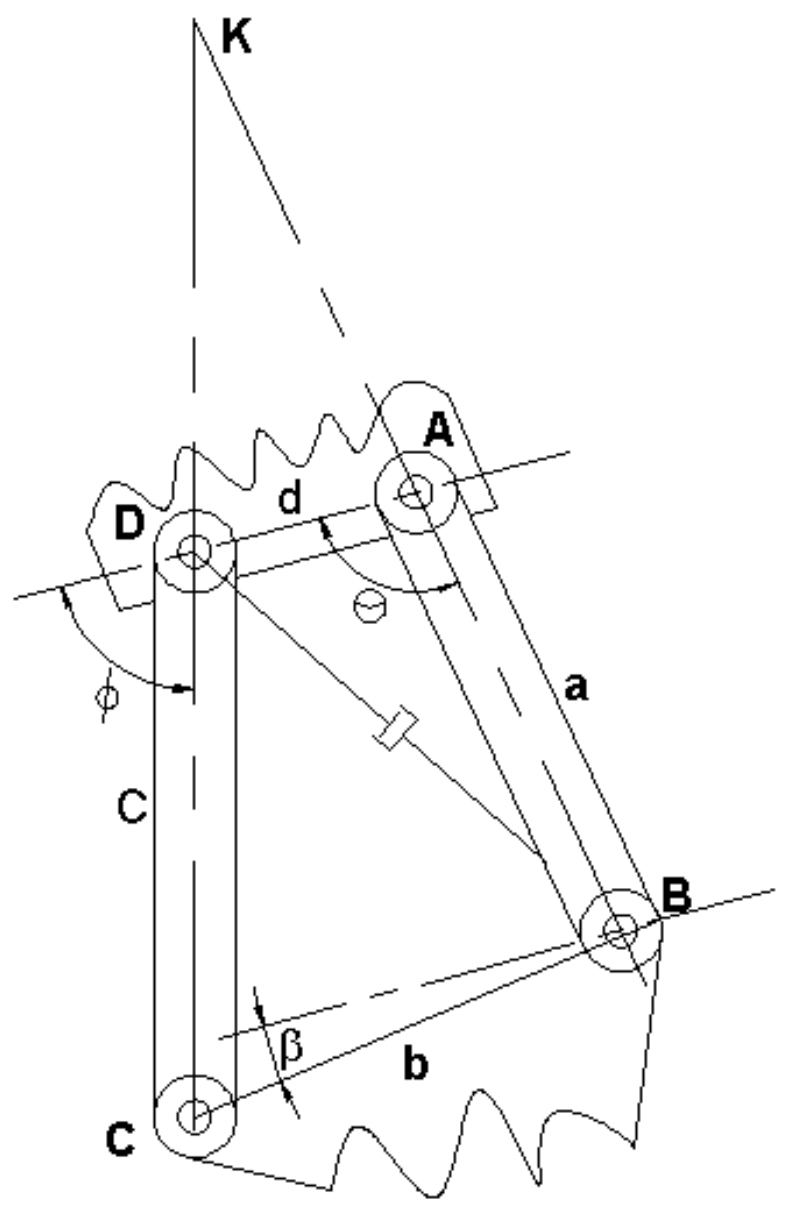

Fig. 3 Four Bar Mechanism.

$$
\begin{aligned}
& A_{3}=k_{1}+k_{3}-\left(1+k_{2}\right) \cos \theta \\
& A_{2}=-2 \sin \theta \\
& A_{1}=\left(1-k_{2}\right) \cos \theta+k_{3}-k_{1} \\
& k_{4}=\frac{d}{b} \\
& \varphi=2 \tan ^{-1}\left(\left(-A_{2} \pm \sqrt{A_{2}^{2}-4 A_{1} A_{3}}\right) / 2 A_{1}\right)
\end{aligned}
$$




$$
\begin{aligned}
& k_{5}=\frac{c^{2}-a^{2}-b^{2}-d^{2}}{2 a b} \\
& A_{5}=\left(k_{4}-1\right) \cos \theta+k_{5}+k_{1} \\
& \beta=2 \tan ^{-1}\left(\left(-A_{2} \pm \sqrt{A_{2}^{2}-4 A_{4} A_{5}}\right) / 2 A_{4}\right) \\
& A_{4}=\left(1+k_{4}\right) \cos \theta+k_{5}-k_{1} \\
& \omega_{2}=\frac{-a \omega_{1} \sin (\phi-\theta)}{b \sin (\phi-\beta)} \quad \omega_{3}=\frac{-a \omega_{1} \sin (\beta-\theta)}{c \sin (\phi-\beta)} \\
& \text { (5) } \alpha_{2}=\frac{-a \alpha_{1} \sin (\phi-\theta)+a \omega_{1}^{2} \cos (\phi-\theta)+b \omega_{2}^{2} \cos (\phi-\beta)-c \omega_{3}^{2}}{b \sin (\phi-\beta)} \\
& \alpha_{3}=\frac{-a \alpha_{1} \sin (\beta-\theta)+a \omega_{1}^{2} \cos (\beta-\theta)+b \omega_{2}^{2}-c \omega_{3}^{2} \cos (\phi-\beta)}{c \sin (\phi-\beta)}
\end{aligned}
$$

Take, $\quad \mathrm{a}=60 \mathrm{~mm} \quad \mathrm{~b}=46.6 \mathrm{~mm} \quad \mathrm{c}=65 \mathrm{~mm} \quad \mathrm{~d}=33.3 \mathrm{~mm}$ The flexion angle of knee during swing phase can be obtained according to the analysis shown in Figure (4)

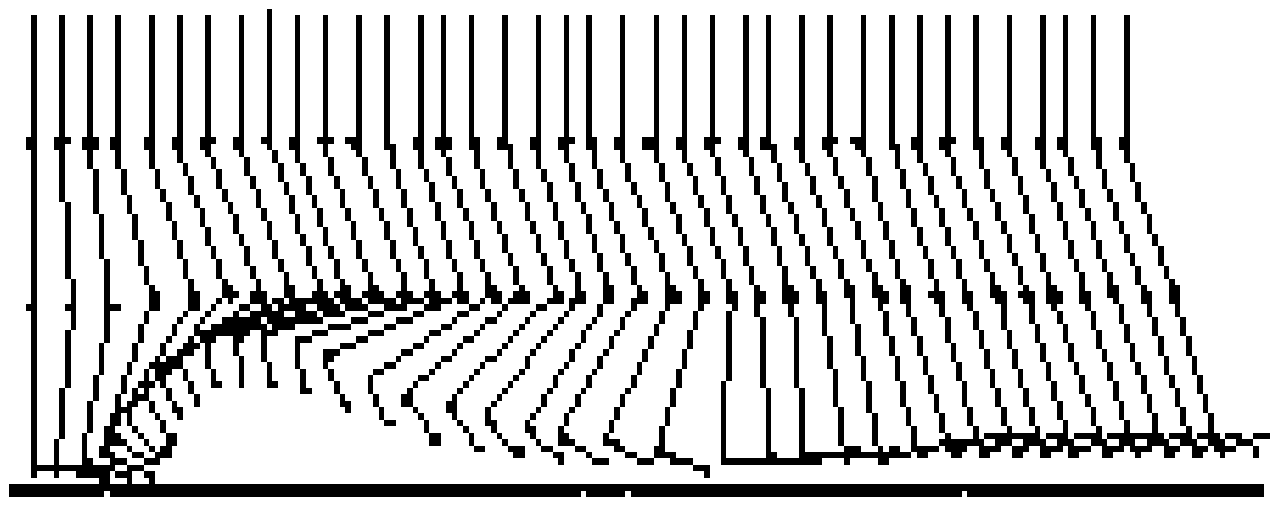

Fig.4 Variation of Flexion Angle of Knee during Walking [4]

Figures (5) and (6) represents the variations of velocity and acceleration of link $\mathrm{CD}$ during walking (stance phase to maximum knee flexion). These variations were studied at different values of shank angular velocities (- $0.35,-0.65,-1 \mathrm{rad} / \mathrm{s})$. These values of angular velocity represented slow, medium and fast walking. Also these variations were studied when the human body moved with variable velocity. 


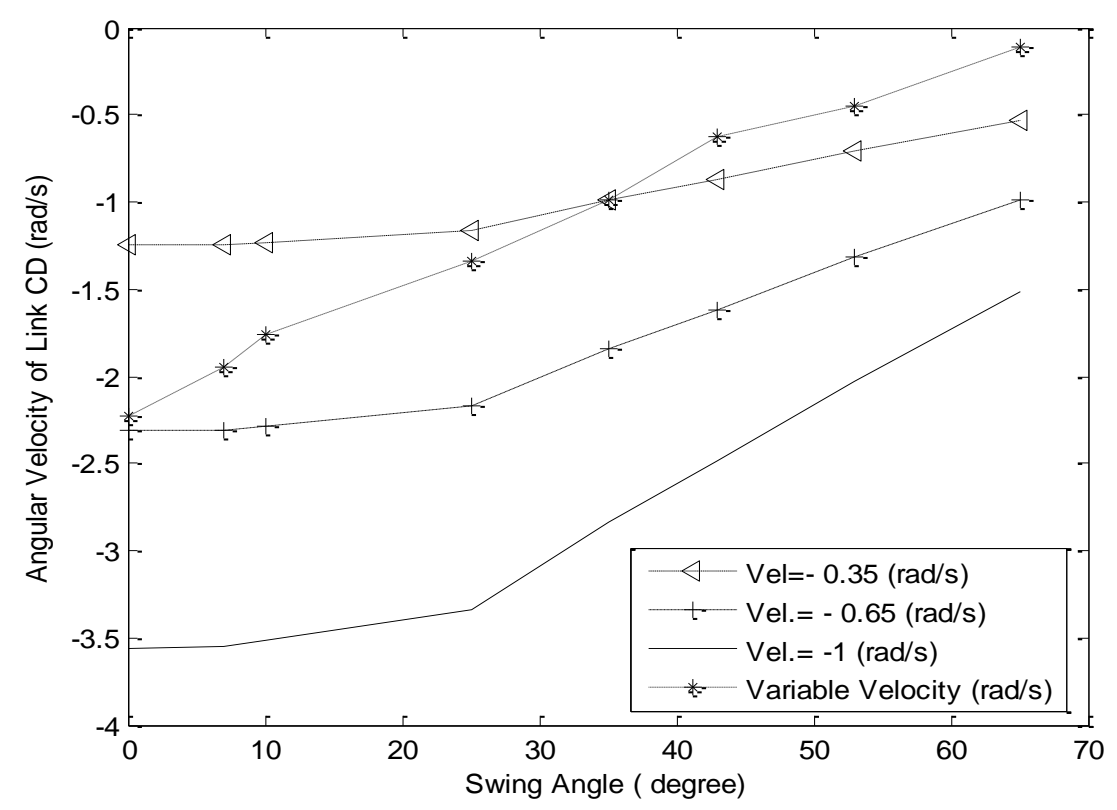

Fig.5 Angular Velocity of Link CD versus Swing Angle

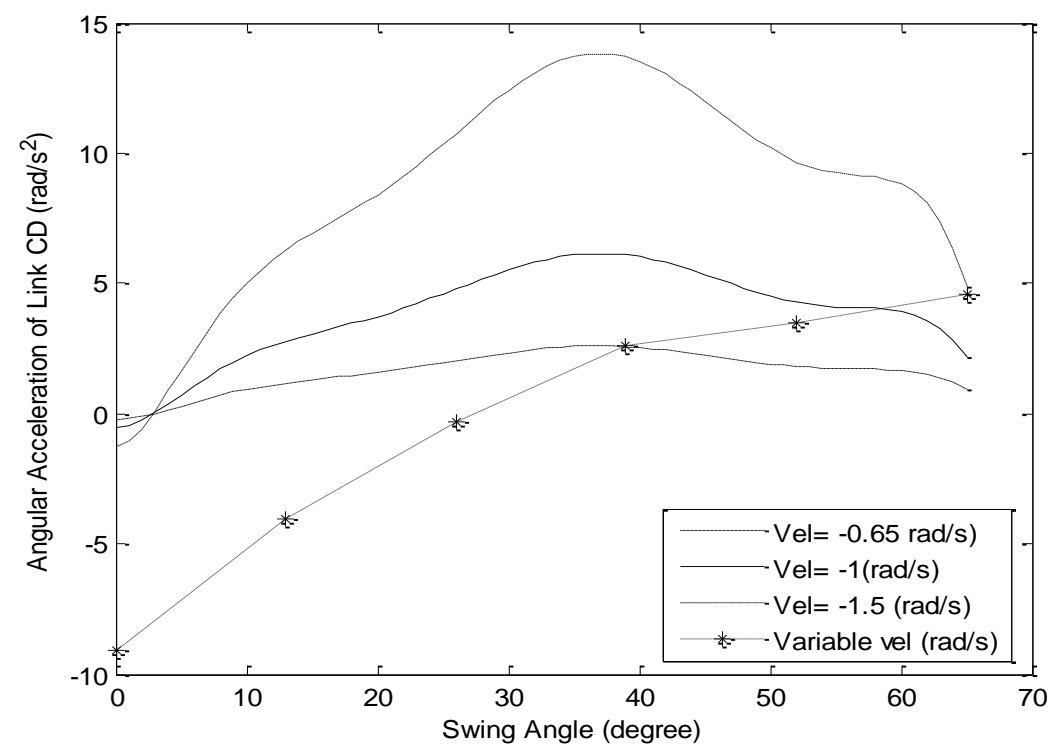

Fig. 6 Angular Acceleration of Link CD versus Swing Angle

The variation of shank acceleration $\ddot{S}$ during the (stance phase and maximum knee flexion) is shown in Figure (7). 


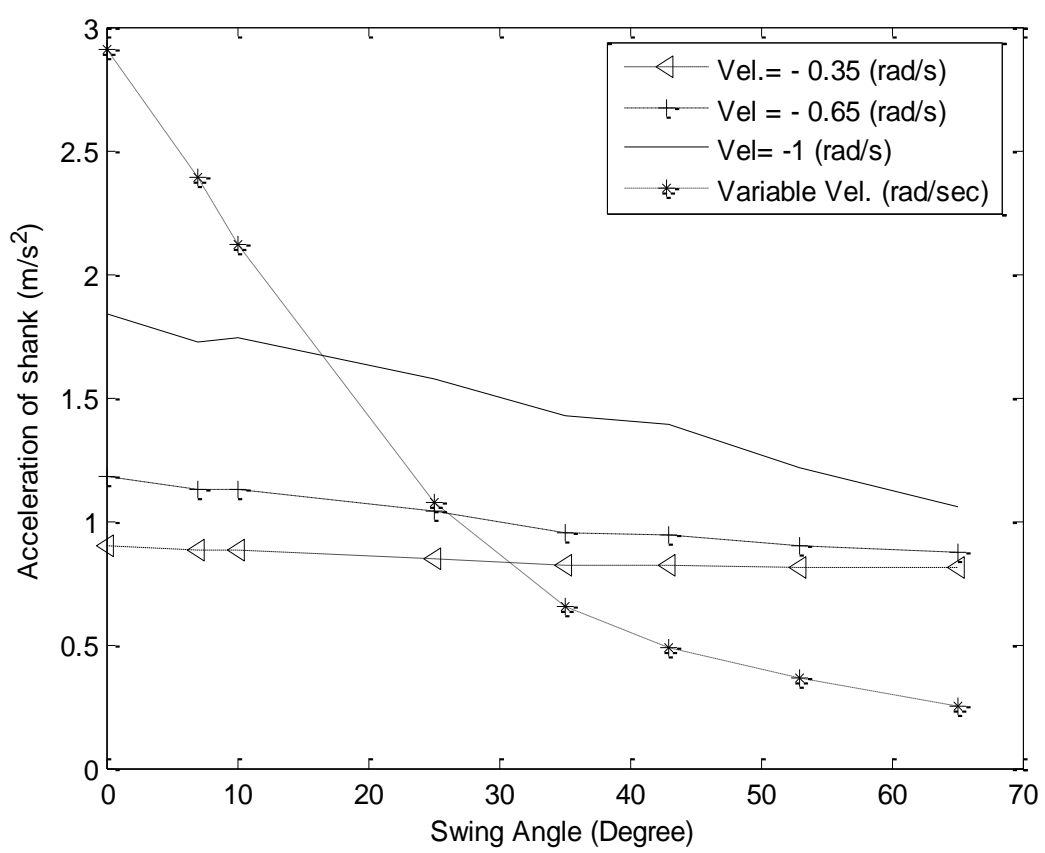

Fig. 7 Acceleration of Shank $\ddot{S}$ versus Swing Angle

\section{HYDRAULIC CONTROL UNIT OF ARTIFICIAL KNEE}

To realize the stability of artificial knee during walking, a hydraulic control unit (damper) is required to be fixed in the four bar mechanism, and a special (ER) damper model must be selected. A control force (damping force) is to be calculated by this model as follows:

$\mathrm{F}_{C}=\mathrm{K}_{\mathrm{e}}\left(\mathrm{Z}_{\mathrm{u}}-\mathrm{Z}_{\mathrm{r}}\right)+\mathrm{C}_{\mathrm{e}}\left(\dot{\mathrm{Z}}_{\mathrm{u}}-\dot{\mathrm{Z}}_{\mathrm{r}}\right)+\mathrm{F}_{\mathrm{er}} \operatorname{sign}\left(\dot{\mathrm{Z}}_{\mathrm{u}}-\dot{\mathrm{Z}}_{\mathrm{r}}\right)$

Where:

$\mathrm{K}_{\mathrm{e}}=\frac{\mathrm{A}_{\mathrm{r}}{ }^{2}}{\mathrm{C}_{\mathrm{g}}}=$ the equivalent stiffness of ER fluid. $\quad \mathrm{R}_{\mathrm{e}}=\frac{12 \eta \mathrm{L}}{\mathrm{b}_{\mathrm{w}} \mathrm{h}^{3}}=$ the fluid resistance.

$\mathrm{C}_{\mathrm{g}}=\frac{\mathrm{V}_{\mathrm{o}}}{\mathrm{P}_{\mathrm{o}} \mathrm{k}_{\mathrm{d}}} \quad . \mathrm{C}_{\mathrm{e}}=\left(\mathrm{A}_{\mathrm{p}}-\mathrm{A}_{\mathrm{r}}\right)^{2} \mathrm{R}_{\mathrm{e}}=$ equivalent damping of the ER fluid.

$\left(\mathrm{Z}_{\mathrm{u}}, \mathrm{Z}_{\mathrm{r}}\right)=$ upper and rear excitations displacement of damper piston. (m)

$\left(\dot{\mathrm{Z}}_{\mathrm{u}}, \dot{\mathrm{Z}}_{\mathrm{r}}\right)=$ upper and rear excitation velocity of damper piston. $(\mathrm{m} / \mathrm{s})$

$$
\mathrm{F}_{\mathrm{er}}=\left(\mathrm{A}_{\mathrm{p}}-\mathrm{A}_{\mathrm{r}}\right) \mathrm{P}_{\mathrm{er}} \quad \mathrm{P}_{\mathrm{er}}=\frac{2 \mathrm{~L} \alpha \mathrm{E}^{\gamma}}{\mathrm{h}}
$$

Table 1 shows the different values of ER damper parameters for the selected model. 
Table (1) ER damper parameters [15]

\begin{tabular}{|l|c|c|}
\hline \multicolumn{1}{|c|}{ Parameters } & Value & Units \\
\hline Initial volume of gas chamber $\left(\mathrm{V}_{o}\right)$ & $25 \times 10^{-6}$ & $m^{3}$ \\
\hline Initial pressure of gas chamber $\left(\mathrm{P}_{o}\right)$ & 25 & $\mathrm{~Pa}$ \\
\hline Specific heat ratio $\left(\mathrm{k}_{\mathrm{d}}\right)$. & 1.4 & --- \\
\hline Piston rod area $\left(\mathrm{A}_{\mathrm{r}}\right)$. & 0.00025 & $\mathrm{~m}^{2}$ \\
\hline Piston area $\left(\mathrm{A}_{\mathrm{p}}\right)$. & 0.00071 & $m^{2}$ \\
\hline Electrode length $(\mathrm{L})$ & 0.258 & $\mathrm{~m}$ \\
\hline Electrode width $\left(\mathrm{b}_{\mathrm{w}}\right)$ & 0.01 & $\mathrm{~m}$ \\
\hline Electrode gap $(\mathrm{h})$ & 0.00075 & $\mathrm{~m}$ \\
\hline Fluid viscosity $(\eta)$ for ER -100 at 60 degree $\mathrm{C}$ & 120 & $\mathrm{mPa}$ \\
\hline Intrinsic value of ER fluid $(\gamma)$ & 1.42 & --- \\
\hline Intrinsic value of ER fluid $(\alpha)$ & 0.8 & --- \\
\hline Value of e.m.f $(\mathrm{E})$ & $0-4$ & $\mathrm{~V}$ \\
\hline
\end{tabular}

\section{Results and Discussion}

Referring to equation (1) the stability moment $\left(\mathrm{M}_{\mathrm{B}}\right)$ for the artificial leg during walking can be obtained, knowing the following:-

1. The control (damping) force $\mathrm{F}_{\mathrm{c}}$ can be obtained from equation (6).

2. The acceleration of shank $\ddot{S}$ and angular acceleration can determined from analysis of the four bar mechanism.

3. The flexion angle of knee during swing phase can be obtained from the analysis of human walking shown in Figure (4)

4. Moment of inertia and mass of shank and its dimensions are obtained from Table (2),

Table (2) Moment of inertia and mass of the shank and its dimensions [11, 16, 17]

\begin{tabular}{|c|c|c|c|}
\hline Segment & Mass $(\mathrm{kg})$ & $\begin{array}{c}\text { Mass moment of } \\
\left.\text { Inertia( } \mathrm{kg} \mathrm{m}^{2}\right)\end{array}$ & $\begin{array}{c}\text { C.G location } \\
\text { \% Seg. Length }\end{array}$ \\
\hline Foot & 1.068 & $0.987 * 10^{-2}$ & 0.35 \\
\hline Shank & 2.884 & $10^{-2} 3.167 *$ & 0.433 \\
\hline Thigh & 6.516 & $7.402 * 10^{-2}$ & 0.433 \\
\hline
\end{tabular}

Figure (8) describes the relation between damping force and the velocity of damper during swing phase (quadriceps action). 


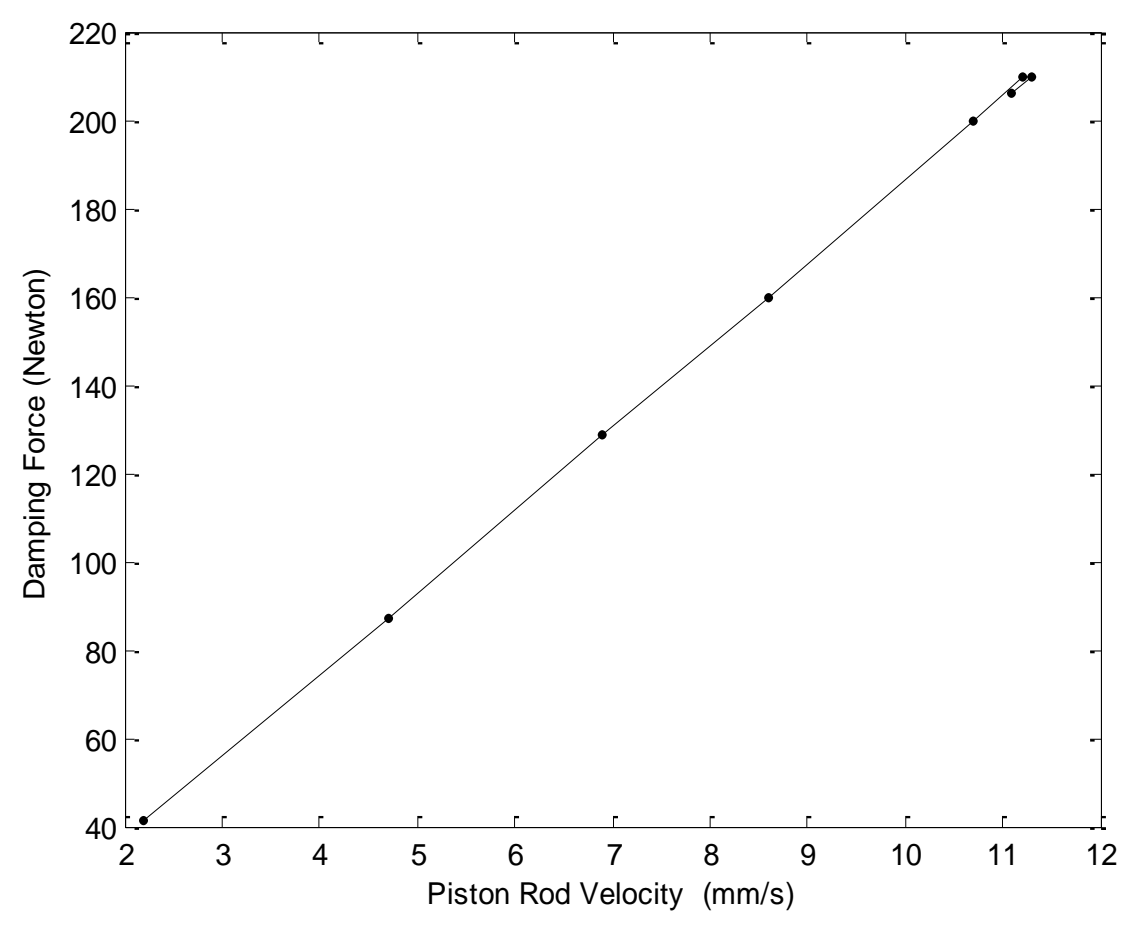

Fig. 8 Damping Force versus Piston Rod Velocity

The variation of damping force during swing phase is shown in Figure (9). This variation was obtained when the human body moves with slow, medium, and fast motion.

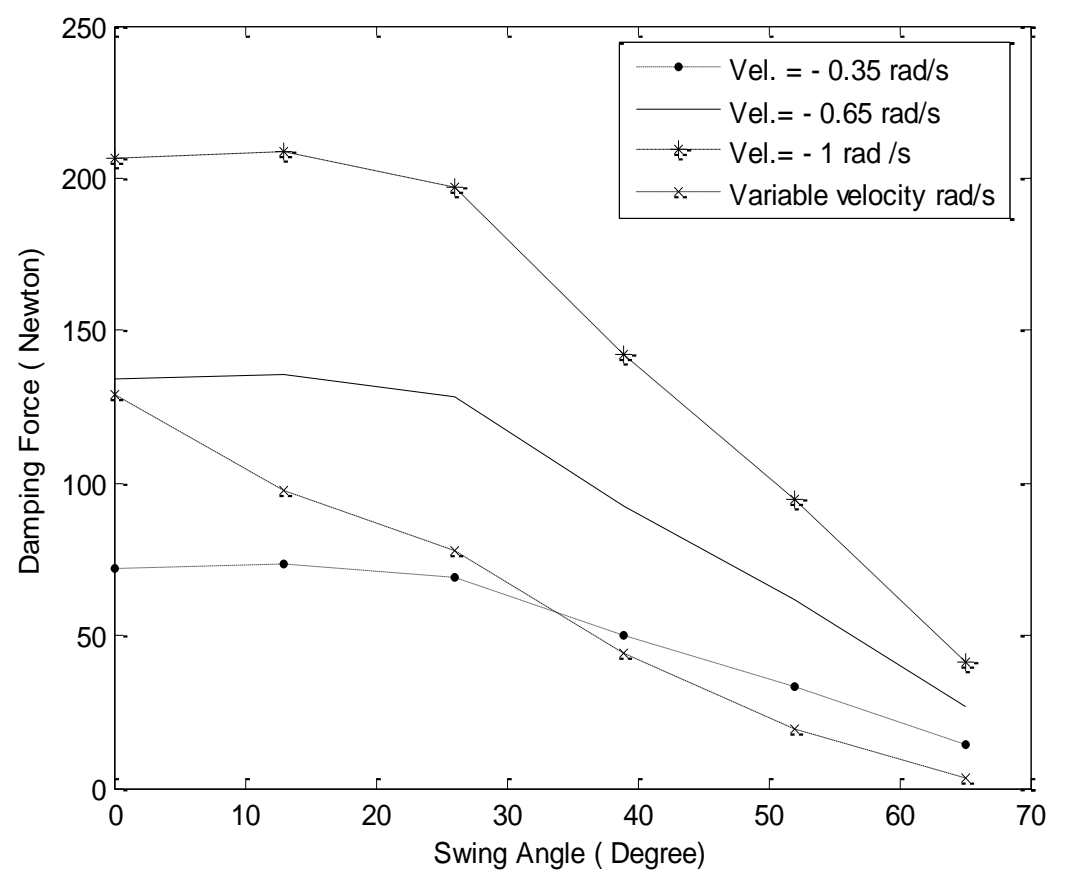

Fig.9 Variation of Damping Force During Walking with Swing Angle 
From this figure it is seen that the compression force decreases throughout a change of swing angle from $0-65^{\circ}$ with a compression ratio of 4.5 in the case of fast walking and 3 for the case of slow walking. Also when the human body moves with variable velocity the damping force is decaying slightly during walking.

Figure (10) demonstrates the stability moment of artificial knee during swing phase for different methods of motion. These are, slow, medium, and fast walking .Also this figure shows the stability moment when the human body moves with variable velocity. It is shown that, when the human body moves with constant velocity the stability moment increases reaches a maximum value then decreases before the end of swing phase. But when the human body moves with variable velocity the stability moment increases to a maximum at the end of swing phase.

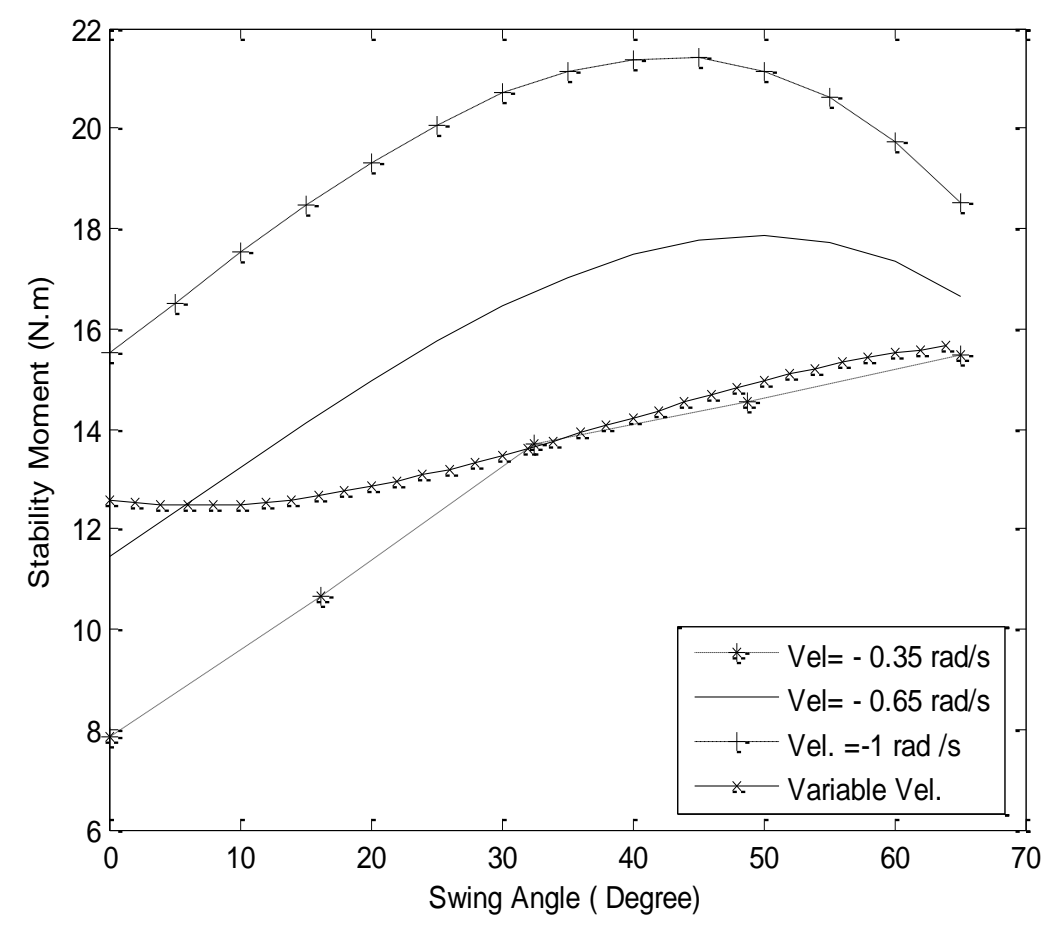

Fig.10 Stability Moment during Slow, Medium, Fast and Variable Velocity Walking

The stability moment of artificial knee between toes -off and maximum knee flexion for different means of control unit is shown in Figure (11). These are, pneumatic swing control (slow and fast walking), hydraulic swing control with linear extension bias [18], normal leg [19], and artificial knee using ER damper (present work).On comparing the patterns of variation, it is clear that the values of stability moment in the case of pneumatic and hydraulic swing control are less than that required for normal human walking. (ER damper) as a control unit in artificial knee gives more stability for artificial leg similar to that of normal walking. This means that using ER fluid damper is more realistic than the other classical types 


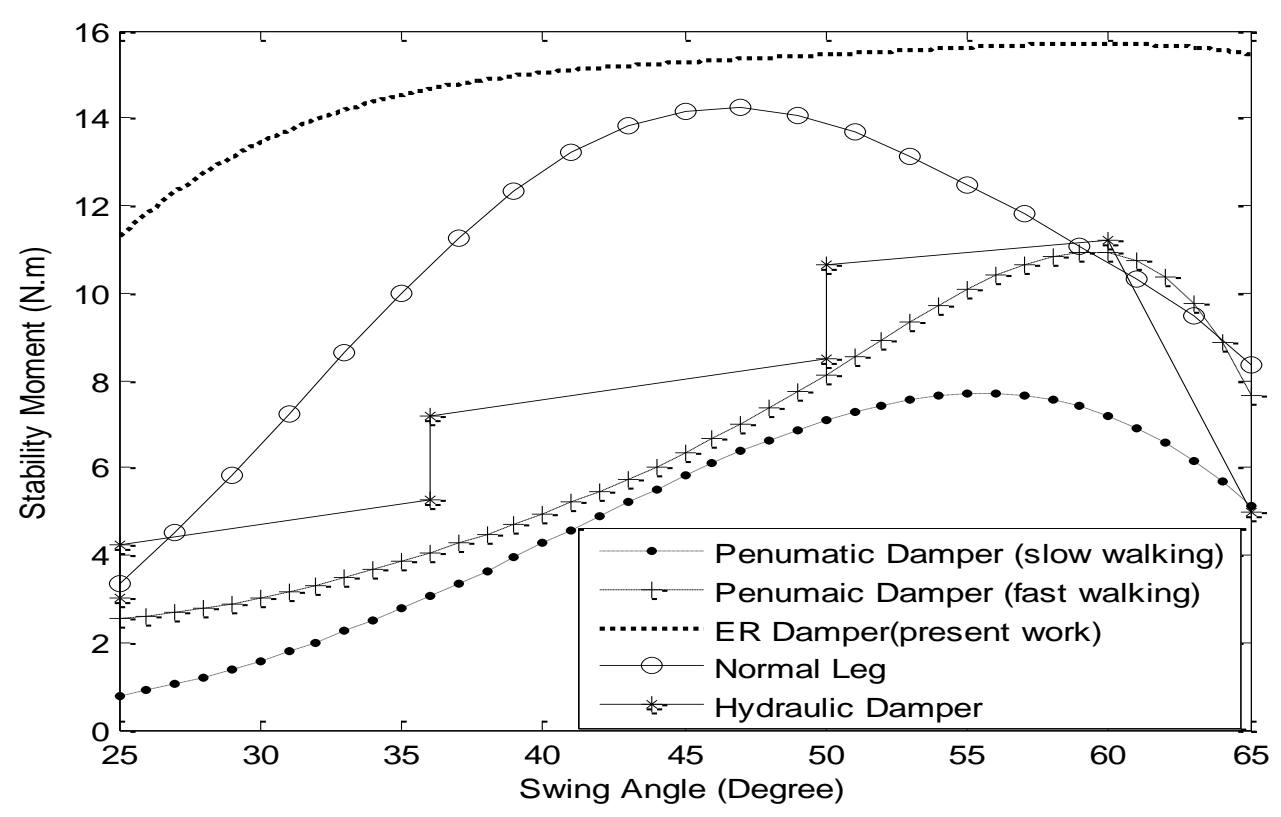

Fig.11 Prosthetic Knee Moment for Different Method of Motion Control

\section{CONCLUSIONS}

Several factors are involved in selecting the most appropriate knee for a particular patient. When deciding between single-axis knee and a four-bar linkage knee using ER fluid damper these factors include stance-phase stability, swing-phase control, and weight of the knee unit, durability and cost. The final conclusions, drawn from present study, can be summarized as follows:

1- ER damper used for artificial knee is suitable to achieve the stability of knee during walking.

1. The trend of stability moment for artificial knee using ER damper is the same as of stability moment for normal knee during walking.

2. The ER damper used in artificial mechanism is more realistic than the other classical types.

\section{REFERENCES}

1- Appoldt, F.A. and Bennett, L., "A Preliminary Report on Dynamic Socket Pressures", Bulletin of prosthetics Research, New York ,U.S, Fall 67, pp 20-55, 1967.

2- Bielefeldt, A. and Schreck, H.J., 1979 "The Altered Alignment Influence on Above Knee Prosthesis Socket Pressure Distribution", International Series on Biomechanics, VIIa. pp. 387-393. 1981. 
3. Dorious, L.K., "Errors in Alignment of Center of Pressure and Foot Coordinates Affect Predicted Lower Extremity Torques", Journal of Biomechanics, Vol.17, No.1, pp.1-9 1984.

4. Rohrle, H. ,Scholten, R., Sigolotto C., and Sollbach, W. ,'Joint Forces the Human Pelvis - Leg Skeleton During Walking “,Biomechanics, Vol.17,No.6 , pp.409-424, West Germany, 1984.

5. Hunter, G.D., "Obstruction of the Swing Phase during the Gait, Phase -Dependant Bilateral Leg Muscle Coordination”, Brain Research, 384 pp.166-169, 1986.

6. Wen Liu and Nigg, B.M., "A Model of the Neuromuscular - Skeletal System for Human Locomotion”, Biological Cybernetics, 73, pp.97-111, 1995.

7. Nisell, R., "Mechanics of the Knee, A Study of Joint and Muscle Load with Clinical Applications", Acta Orthop. Scand. Suppl., 216, pp. 1-42, 1985.

8. Hatze, S., "An Investigation of Foot-Clearance Issues in Normal and Above-Knee Amputee Gait", PhD. Dissertation, Northwestern University, Evanston, 111, pp.83$141,1957$.

9. Kim, S. and Pandy, M.G., "A Two-Dimensional Dynamic Model of the Human Knee Joint", Biomed. Sci. Instrum., 29, pp. 33-46, 1993.

10. Radcliffe C.W., "A Three-Dimensional Mathematical Model of the Knee-Joint”, J. Biomech., 13, pp. 677-685, 1980.

11. Braune, .W. and Fischer, O., "Bestimmung Der Tragheitsmomente des Menschlichen Korpers und Seiner Glieder", Published by S.Hirzal, Leipzig 1892. "Determination of Body Moments of Inertia of the Human Body and its Limbs", Translators P. Maquet and R.Furlong, Springer-Verlag, 1988.

12. Schede, D., "Manual for the Prosthetic", Orthotic Center of the Northwestern University, Medical School, Chicago, Biomech, 29, pp. 151-160, 1995

13. Zarrugh, M.Y.and Radcliffe, C.W.," simulation of swing phase dynamics in aboveknee prostheses",j. Biomechanics, Vol. 9 pp.283-292,1976.

14. Khurmi and Gupta," Computer Aided Analysis and Synthesis of Mechanisms “, A text book, Eyrasia Publishing House (PVT.) LTD. Ram Nagar, New Delhi 110055, 1996

15. Choi, S.B., Choi, Y.T. and park, D.W.,"A Sliding Mode Control of Fully Car Electro-rheological Suspension System Via Hardware in Loop", Simulation Journal of Dynamic Systems Measurement and Control Transaction of ASME, Vol. 122, pp.114-121, March 2000 .

16. Winter, D .A. "Kinematics of Normal Locomotion" - A statistical study Based on T.V Data., Journal of Biomechanics, Vol. 7, pp.479-486, 1979.

17. Bresler, B. and Frankel, J.P., "The Forces and Moments in the Leg during Level Walking”, ASME, Vol. 72, pp. 27-36, 1950.

18. Radcliffe, C.W.,"Above-knee prosthetics", The knud Jansen lecture, New- York , 1977, Department of Mechanical Engineering, University of California , Berkely.

19. Davy, D.T. and Audu, M.L., "Dynamic Optimization Technique for Prediction Muscle Forces in the Swing Phase of Gait", Biomechanics, Vol. 20, No 2, pp. (187-201), 1987. 
نموذج رياضي لرجل صناعية بأستخدام مساعد هيدروليكى يعمل بالمجال الكهربى

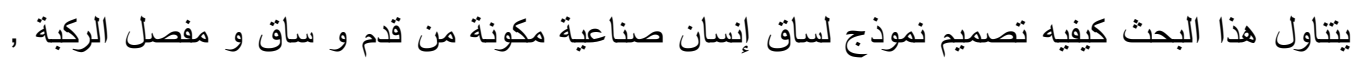

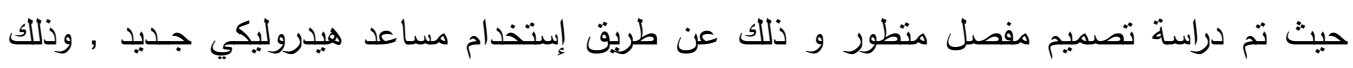

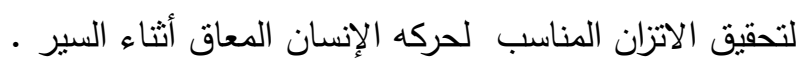

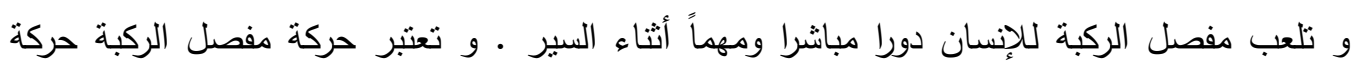

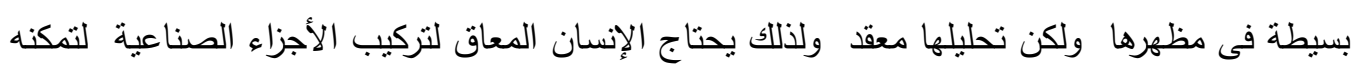
من الحركة في صورة أقرب مايمكن من الحركة الطبيعية .

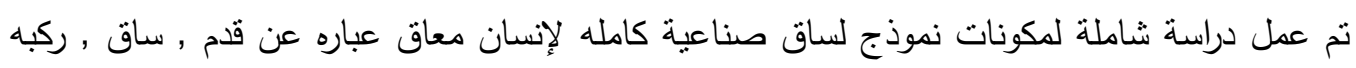

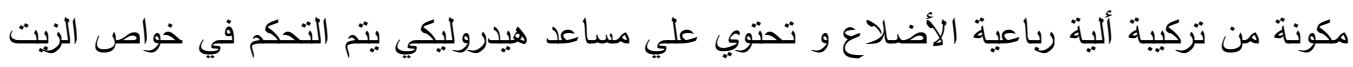

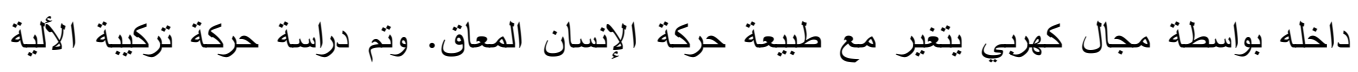

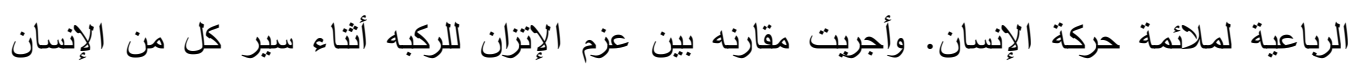

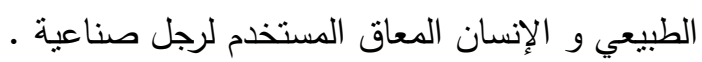

